\title{
Reason for Non-Completion
}

National Cancer Institute

\section{Source}

National Cancer Institute. Reason for Non-Completion. NCI Thesaurus. Code C49627.

The reason that a subject was discontinued from a clinical trial. 